\title{
Fatores associados à masculinidade no diagnóstico precoce do câncer de próstata: revisão narrativa
}

RESUMO | Objetivo: identificar os fatores associados à masculinidade no diagnóstico precoce do câncer de próstata. Método: trata-se de uma revisão narrativa realizada nas seguintes bases de dados: SCIELO, LILACS e PUBMED. Após a aplicação dos critérios de elegibilidade, a amostra do estudo foi composta por 14 artigos publicados entre 2000 e 2021 . Resultados: os estudos abordam fortemente a influência dos aspectos socioeconômicos e culturais na busca preventiva dos homens pelo autocuidado. Os padrões estipulados pela sociedade dificultam a adesão masculina fazendo-os diminuir a procurar por serviços de saúde em relação às mulheres dificultando o diagnóstico precoce do câncer de próstata. O exame de toque retal encontra-se atrelado à transgressão de sua masculinidade repercutindo no medo de realizá-lo. Conclusão: concepções socioculturais acerca da masculinidade associam-se negativamente à saúde dos homens impondo dificuldades no diagnóstico precoce do câncer e, conseqüentemente, aumentando a mortalidade masculina.

Palavras-chaves: Neoplasias da Próstata. Masculinidade. Saúde do Homem. Antígeno Prostático Específico. Exame Retal Digital.

ABSTRACT | Objective: to identify the factors associated with masculinity in the early diagnosis of prostate cancer. Method: this is a narrative review conducted in the following databases: SCIELO, LILACS and PUBMED. After applying the eligibility criteria, the study sample was composed of 14 articles published between 2000 and 2021. Results: the studies strongly address the influence of socioeconomic and cultural aspects on men's preventive search for self-care. The standards set by society hinder male adherence, making them less likely to seek health services than women, hindering the early diagnosis of prostate cancer. The rectal examination is linked to the transgression of their masculinity, resulting in the fear of performing it. Conclusion: sociocultural conceptions about masculinity are negatively associated with men's health, imposing difficulties in the early diagnosis of cancer and consequently increasing male mortality.

Keywords: Prostatic Neoplasms. Masculinity. Men's Health. Prostate-Specific Antigen. Digital Rectal Examination.

RESUMEN | Objetivo: identificar los factores asociados a la masculinidad en el diagnóstico precoz del cáncer de próstata. Método: se trata de una revisión narrativa realizada en las siguientes bases de datos: SCIELO, LILACS y PUBMED. Tras aplicar los criterios de elegibilidad, la muestra del estudio estuvo compuesta por 14 artículos publicados entre 2000 y 2021 . Resultados: los estudios abordan la influencia socioeconómica y cultural en la búsqueda preventiva del autocuidado por los hombres. Las normas estipuladas por la sociedad dificultan la adherencia masculina haciendo que reduzcan la búsqueda de servicios sanitarios en relación con las mujeres dificultando el diagnóstico precoz del cáncer de próstata. El tacto rectal está vinculado a la transgresión de su masculinidad, lo que provoca miedo a realizarlo. Conclusión: las concepciones socioculturales sobre la masculinidad se asocian negativamente con la salud del hombre imponiendo dificultades en el diagnóstico precoz del cáncer y, en consecuencia, aumentando la mortalidad masculina.

Palabras claves: Neoplasias de la Próstata. Masculinidad. Salud del Hombre. Antígeno Prostático Específico. Tacto Rectal.

\section{Karoline Gandra Pereira}

Acadêmica do curso de graduação em Medicina pela Universidade Vale do Rio Doce (UNIVALE). Governador Valadares, $M G$, Brasil.

ORCID: 0000-0003-0622-7495

\section{Samila Maria Pereira de Cristo}

Acadêmica do curso de graduação em Medicina pela Universidade Vale do Rio Doce (UNIVALE). Governador Valadares, MG, Brasil. ORCID: 0000-0002-9133-4473

\section{Fabrícia Josely Oliveira Barbosa}

Enfermeira, Mestre em Gestão de Serviços de Saúde pela Universidade Federal de Minas Gerais (PPGGSS/UFMG). Belo Horizonte, MG, Brasil.

ORCID: 0000-0003-0786-0284

\section{Patrick Leonardo Nogueira da Silva}

Enfermeiro, Mestrando pelo Programa de Pós-Graduação em Cuidado Primário em Saúde da Universidade Estadual de Montes Claros (PPGCPS/UNIMONTES). Montes Claros, MG, Brasil.

ORCID: 0000-0003-2399-9526

\section{Ana Patrícia Fonseca Coelho Galvão}

Enfermeira, Doutoranda em Ciências da Saúde pela Faculdade de Ciências Médicas da Santa Casa de São Paulo (FCMSCSP), Professora do Departamento de Enfermagem da Universidade Ceuma (UNICEUMA). São Luís, MA, Brasil.

ORCID: 0000-0003-3376-5678

\section{Carolina dos Reis Alves}

Enfermeira, Doutora em Ciências da Saúde,
Professora do Departamento de Enfermagem da Faculdade Santo Agostinho (FASA). Montes Claros, MG, Brasil.

ORCID: 0000-0003-2107-6306

Recebido em: 14/04/2021

Aprovado em: 28/04/2021

INTRODUÇão

er homem, nas sociedades oci-
dentais contemporâneas, res-
guardadas as diferenças existentes, é estar enquadrado no ideal de masculinidade. Para alcançar tal ideal, os homens negam comportamentos tidos como femininos e prezam por demonstrar características tidas como masculinas e, categoricamente determinadas 
pela sociedade, tais como a virilidade, a força e a inviolabilidade, sacrificando, muitas vezes, a própria saúde em prol desse ideal ${ }^{(1)}$.

Dessa maneira, há homens que se ausentam na busca pelo cuidado devido ao medo de estarem se afeminando ao buscá-lo, por temerem descobrir algo que comprometa o ideal de força e por sentirem desconfortáveis na exposição ao médico ${ }^{(2)}$. Sendo assim, essas concepções socioculturais atuam normalizando convicções arcaicas do que é "ser homem" e, por conseguinte, comprometem a saúde masculina de maneira que o índice de adoecimento e mortalidade desse grupo populacional seja alto, sobretudo no que tange o Câncer de Próstata (CAP), uma vez que, em inúmeros casos, tem seu diagnóstico tardio ${ }^{(1,2)}$.

A não adesão dos homens na busca pelos serviços de saúde revela estereótipos de gênero baseados em características culturais, que normatizam certo tipo de masculinidade tida como hegemônica, obedecendo a uma ordem simbólica na qual a doença expressa à fragilidade do corpo e, por extensão, do seu portador ${ }^{(3)}$.

Assim, segundo o Instituto Nacional do Câncer (INCA), hodiernamente, no Brasil, o CAP é considerado a segunda causa de óbito devido a neoplasias em homens, estando superado apenas pelo Câncer de Pele Não Melanoma (CPNM). Do mesmo modo, é importante salientar que esse tipo de tumor pode crescer tanto de forma lenta, quanto rápida. Por isso, torna-se evidente a necessidade da realização de exames de rotina para a sua detecção precoce e, assim, possibilitar melhor eficácia do tratamento. Dessa forma, os principais e primeiros exames realizados devem ser o de toque retal e a dosagem do Prostate Specific Antigen (PSA) ${ }^{(4)}$.

Com o exame do toque retal, é possível verificar a presença de nódulos na glândula, avaliando tamanho, consistência e forma da próstata ${ }^{(4)}$, enquanto que o PSA é o marcador sérico mais utilizado clinicamente em relação ao CAP e seu uso engloba não somente o rastreamento, como também o diagnóstico, prognóstico e monitoramento da terapia ${ }^{(5)}$. Além disso, especialistas sugerem que, homens a partir dos 50 anos precisam realizar o PSA e o toque retal anualmente. Entretanto, indivíduos com histórico familiar de CAP devem realizar tais procedimentos a partir dos 40 anos $^{(3-5)}$. Destarte, debater sobre esse assunto é extremamente relevante na área da saúde masculina e investigar sobre essa temática é um exercício de ampliação do conhecimento de acadêmicos de medicina.

Sendo assim, este estudo objetivou identificar os fatores associados à masculinidade no diagnóstico precoce do CAP.

\section{MÉTODO}

Trata-se de um estudo descritivo, exploratório, documental, retrospectivo, com base em uma revisão narrativa da literatura. A busca pelos artigos foi realizada por meio das bases de dados online da Biblioteca Virtual de Saúde (BVS), sendo elas: Scientific Eletronic Library Online (SCIELO), Literatura Latino-Americana e do Caribe em Ciências da Saúde (LILACS) e Medical Literature Analysis and Retrieval System Online (MEDLINE/PUBMED). A consulta a estes bancos de dados foi tida como critério inicial para seleção. Optou-se por estas bases de dados em decorrência destas serem as principais fontes de publicações científicas na atualidade e, a partir de seu sistema de busca, foram utilizados os seguintes descritores: "CAP"; "masculinidade"; "saúde do homem"; "antígeno prostático específico" e "exame retal digital". Ainda, os mesmos foram combinados entre si pelo operador booleano "AND" no intuito de definir relações entre os termos em uma pesquisa, ou seja, os termos são combinados para que cada resultado contenha no mínimo um dos termos.

Foram adotados os seguintes critérios de inclusão para a captação dos artigos:
(1) estar indexado nas respectivas bases de dados; (2) ter o resumo disponível para leitura na língua portuguesa; (3) estar disponível gratuitamente para download; (4) ser artigo nacional ou internacional; e (5) estar publicado entre o período de janeiro de 2000 a março de 2021. Foram excluídos: (1) os artigos repetidos que se encontravam indexados em mais de uma base de dados; (2) artigos indexados em outras bases de dados diferentes das selecionadas; e (3) os artigos que não abordavam a temática proposta. A busca resultou em uma amostragem de 526 artigos (Tabela 1), sendo que, após a aplicação dos critérios de elegibilidade do estudo, a amostra final foi composta por 14 artigos publicados entre os anos de 2000 e 2021 (Tabela 2). Ainda, foi realizada uma leitura cuidadosa de todos os artigos selecionados a fim de garantir a aplicação dos critérios de elegibilidade. A coleta de dados ocorreu durante o $1^{\circ}$ semestre de 2021, entre os meses de janeiro a março do respectivo ano, pelo pesquisador responsável. Para o mesmo, foi utilizado um formulário estruturado como instrumento de coleta de dados.

A discussão foi estruturada conforme as seguintes categorias: (1) A construção do "homem ideal"; (2) A próstata e o câncer: importância da prevenção; (3) Principais métodos diagnósticos: PSA e exame de toque retal; (4) CAP: conduta médica e tratamento; e (5) Repercussões do CAP na masculinidade. Em suma, o estudo discutiu sobre o ideal de masculinidade presente no Brasil e como isso interfere na saúde do homem, salientando as justificativas do público masculino para a ausência da busca pelos sistemas de saúde. Em seguida, foi argumentado sobre o CAP, evidenciando as formas de diagnóstico, prevenção e tratamento e, por fim, abordaram-se as conseqüências da concepção ultrapassada de masculinidade ainda presente na sociedade contemporânea que interfere e, em muitos casos, impossibilita o diagnóstico precoce da neoplasia prostática. 


\begin{tabular}{|c|c|c|c|c|c|c|c|c|}
\hline \multirow{2}{*}{ Descritores } & \multicolumn{2}{|c|}{ SCIELO } & \multicolumn{2}{|c|}{ LILACS } & \multicolumn{2}{|c|}{ PUBMED } & \multicolumn{2}{|c|}{ TOTAL } \\
\hline & $\mathbf{n}$ & $\%$ & $\mathbf{n}$ & $\%$ & $\mathbf{n}$ & $\%$ & $\mathbf{n}$ & $\%$ \\
\hline "CAP" AND "Masculinidade" & 02 & 0,3 & 14 & 2,9 & 02 & 0,3 & 18 & 3,5 \\
\hline "CAP" AND "Saúde do homem" & 00 & 0,0 & 34 & 6,5 & 02 & 0,3 & 36 & 6,8 \\
\hline "CAP" AND "PSA" & 01 & 0,2 & 333 & 63,3 & 79 & 15,0 & 413 & 78,5 \\
\hline "CAP" AND "exame retal digital" & 00 & 0,0 & 33 & 6,0 & 26 & 5,2 & 59 & 11,2 \\
\hline Total & 03 & 0,5 & 414 & 78,7 & 109 & 20,8 & 526 & 100,0 \\
\hline
\end{tabular}

Fonte: Autoria própria, 2021.

Tabela 2 - Perfil da amostra após aplicação dos critérios de elegibilidade.

\begin{tabular}{lcccccccccccccc}
\multicolumn{1}{c}{ Descritores } & \multicolumn{1}{c}{ SCIELO } & \multicolumn{1}{c}{ LILACS } & \multicolumn{11}{c}{ PUBMED } & \multicolumn{1}{c}{ TOTAL } \\
& AS & AE & AU & AS & AE & AU & AS & AE & AU & AS & AE & AU \\
"CAP" AND "Masculinidade" & 02 & 00 & 02 & 14 & 09 & 05 & 02 & 02 & 00 & 18 & 11 & 07 \\
\hline "CAP" AND "Saúde do homem" & 00 & 00 & 00 & 34 & 34 & 00 & 02 & 01 & 01 & 36 & 35 & 01 \\
"CAP" AND "PSA" & 01 & 01 & 00 & 333 & 332 & 01 & 79 & 78 & 01 & 413 & 411 & 02 \\
\hline "CAP" AND "Exame retal digital" & 00 & 00 & 00 & 33 & 31 & 02 & 26 & 24 & 02 & 59 & 55 & 04 \\
\hline Total & $\mathbf{0 3}$ & $\mathbf{0 1}$ & $\mathbf{0 2}$ & $\mathbf{4 1 4}$ & $\mathbf{4 0 6}$ & $\mathbf{0 8}$ & $\mathbf{1 0 9}$ & $\mathbf{1 0 5}$ & $\mathbf{0 4}$ & $\mathbf{5 2 6}$ & $\mathbf{5 1 2}$ & $\mathbf{1 4}$
\end{tabular}

Fonte: Autoria própria, 2021.

RESULTADOS

A amostra deste estudo, após seleção nas bases de dados (SCIELO, LILACS e MEDLINE/PUBMED) e aplicação dos cri- térios de elegibilidade, foi representada em uma tabela conforme as seguintes variáveis: título, autor, ano de publicação, periódico, objetivo, método e nível de evidência científica (NE).
DISCUSSÃO

A construção do "homem ideal"

O "homem ideal" é, numa concepção hegemônica, o que se enquadra na

Tabela 3 - Apresentação da amostra do estudo, segundo o título, autor, ano, periódico, objetivo, método e NE, após seleção nas bases de dados (SCIELO, LILACS e MEDLINE/PUBMED) e aplicação dos critérios de elegibilidade. (n=14)

\begin{tabular}{|c|c|c|c|c|c|c|c|}
\hline $\mathrm{N}^{\circ}$ & Título & Autor & Ano & Periódico & Objetivo & Método & NE \\
\hline 1 & $\begin{array}{l}\text { As arranhaduras da mas- } \\
\text { culinidade: uma discussão } \\
\text { sobre o toque retal como } \\
\text { medida de prevenção do } \\
\text { câncer prostático }\end{array}$ & $\begin{array}{l}\text { Gomes, Nasci- } \\
\text { mento, Rebello e } \\
\text { Araújo }^{(6)}\end{array}$ & 2008 & $\begin{array}{l}\text { Cienc. Saú- } \\
\text { de Colet. }\end{array}$ & $\begin{array}{l}\text { Analisar os sentidos atribuídos ao } \\
\text { toque retal, buscando refletir acerca } \\
\text { de questões subjacentes a falas mas- } \\
\text { culinas a partir de aspectos do modelo } \\
\text { hegemônico de masculinidade. }\end{array}$ & $\begin{array}{l}\text { Estudo obser- } \\
\text { vacional com } \\
\text { abordagem } \\
\text { qualitativa }\end{array}$ & $2 C$ \\
\hline 2 & $\begin{array}{l}\text { A prevenção do câncer de } \\
\text { próstata: uma revisão da } \\
\text { literatura }\end{array}$ & $\begin{array}{l}\text { Gomes, Rebello, } \\
\text { Araújo e Nasci- } \\
\text { mento }^{(7)}\end{array}$ & 2008 & $\begin{array}{l}\text { Cienc. Saú- } \\
\text { de Colet. }\end{array}$ & $\begin{array}{l}\text { Analisar as recomendações voltadas } \\
\text { para a prevenção do CAP presentes na } \\
\text { literatura específica sobre o assunto }\end{array}$ & $\begin{array}{c}\text { Revisão da } \\
\text { literatura }\end{array}$ & $1^{\mathrm{A}}$ \\
\hline 3 & $\begin{array}{l}\text { Um toque na masculinida- } \\
\text { de: prevenção do câncer } \\
\text { de próstata em gaúchos } \\
\text { tradicionalistas }\end{array}$ & $\begin{array}{c}\text { Souza, Silva e } \\
\text { Pinheiro }^{(8)}\end{array}$ & 2011 & $\begin{array}{l}\text { Rev. Gaucha } \\
\text { Enferm. }\end{array}$ & $\begin{array}{c}\text { Verificar a adesão dos homens ao } \\
\text { exame de toque retal e analisar as } \\
\text { variáveis que influenciam na sua } \\
\text { adesão. }\end{array}$ & $\begin{array}{l}\text { Estudo transver- } \\
\text { sal com aborda- } \\
\text { gem quantitativa }\end{array}$ & $2 B$ \\
\hline
\end{tabular}

Identidade do homem resiliente no contexto do

4 doente com câncer de próstata: uma perspectiva cultural
Pinto, Muniz,

Schwartz, Budó Lange $^{(9)}$
Rev. Bras. Compreender o contexto do homem

Enferm. resiliente quando doente com CAP.
Estudo de caso etnográfico 


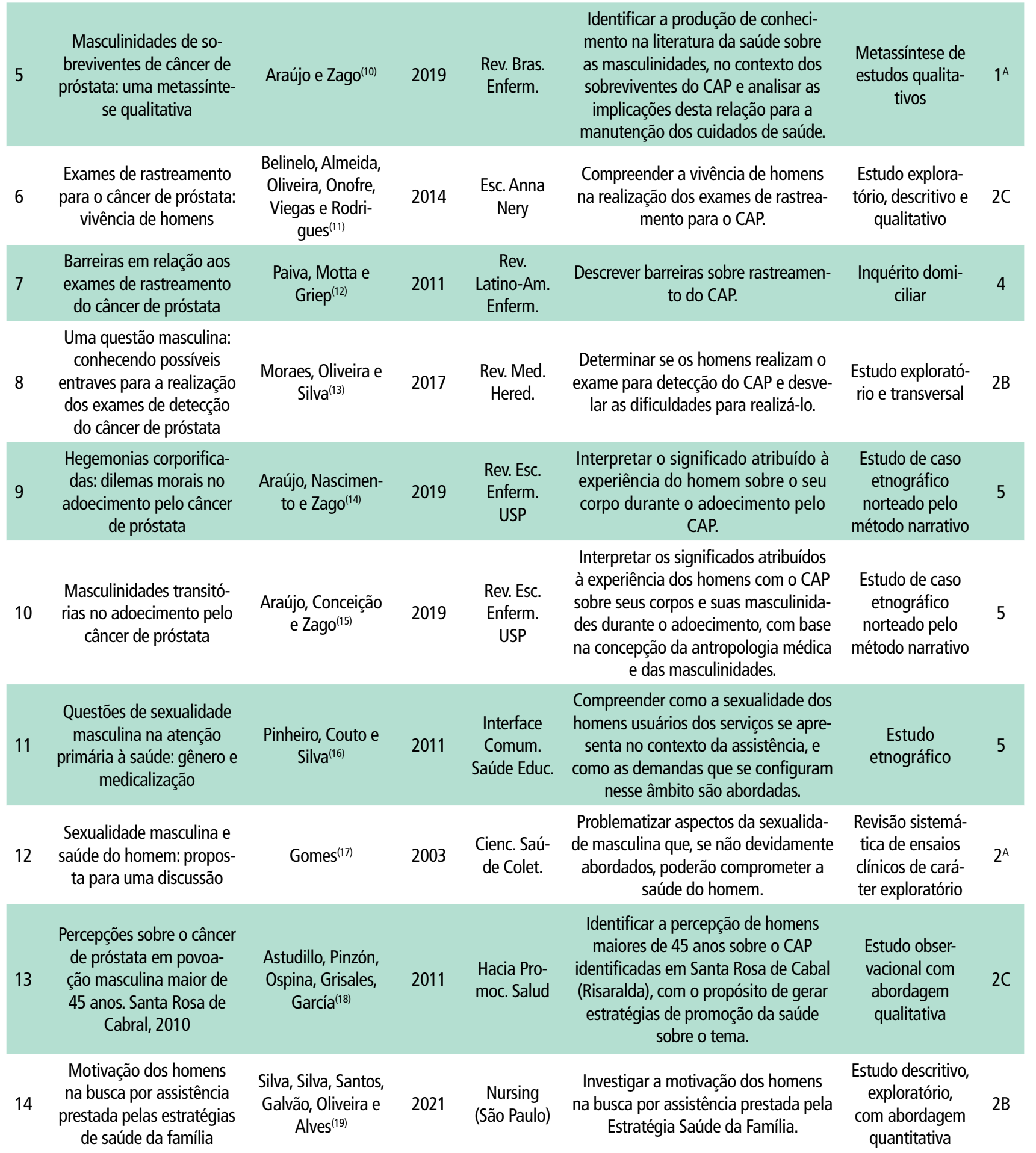

Fonte: Autoria própria, 2021. NE = Nível de Evidência.

masculinidade tida como perfeita a ser seguida, tal ideal foi construído ao longo dos anos numa tentativa de estabelecer traços e ações representativos de uma condição masculina em contraste ao oposto, mulheres, e ao inverso, homosse- xuais $^{(6,14,20)}$. Entre os padrões de masculinidade vigentes, há os mais valorizados e exaltados e outros mais subordinados ${ }^{(21)}$. 
No modelo da masculinidade hegemônica, destacam-se como eixos estruturantes a dominação e a heterossexualida$\mathrm{de}^{(6-8,14,20)}$. Assim, as pessoas que seguem tais padrões aceitos de masculinidade são atestadas como homens e não são questionados pelos que compartilham os mesmos símbolos ${ }^{(7,8,21)}$.

Tal ideal foi construído historicamente com a imagem do homem como o responsável pela família, autor de decisões políticas, detentor de maior acesso ao conhecimento e até como modelo de perfeição representado na anatomia em obras de Da Vinci e Michelangelo, nas pinturas, esboços e esculturas. Desse modo, papéis atribuídos aos seres de sexo masculino ao longo dos anos contribuíram para formação do ideal de masculinidade que enaltece o homem como ser forte, viril e inviolável ${ }^{(2,13,14,16,17,19)}$.

Assim sendo, a partir dessa construção sociocultural de masculinidade, os homens que tentam segui-la acabam, muitas vezes, deixando a saúde como algo não prioritário. Tal fator pode ser exemplificado pelo medo dos homens se sentirem afeminados ao buscarem os serviços de saúde, visto que a busca pelo cuidado é algo considerado do público feminino, isso se justificaria pela socialização que as mulheres recebem, desde cedo, para reproduzirem e consolidarem os papéis que as tornam responsáveis, quase que exclusivamente, pela manutenção das relações sociais, comprometendo-se com o cuidado ${ }^{(2,3,14,19)}$. Outro ponto a ser discutido é a repulsa do público do sexo masculino em se exporem ao médico, justificando que mostrar parte do corpo ou ser tocado em um procedimento colocaria sua masculinidade em risco ${ }^{(6-8,13-17)}$.

Ademais, considerar o homem como o responsável e mantedor financeiro da família contribui para ausência nos serviços de saúde visto que os horários da jornada de trabalho geralmente coincidem com os horários de atendimento, principalmente nas unidades de Atenção Primária a Saúde (APS) ${ }^{(13,14,17-19)}$. Para o homem cuidar da sua saúde, o mesmo teria que se ausentar do seu serviço para tal ação, de modo que isso repercutiria na sua renda familiar, bem como em ônus para o setor em que desempenha a função, tornando-se difícil a sua busca por assistência à saúde ${ }^{(6,19)}$. Por fim, outro fator que repele os homens dos sistemas de saúde é o medo de descobrirem algo grave que os possa levar a uma sensação de fragilidade, comprometendo o ideal de força. Para os homens, é um temor terrível encarar qualquer possibilidade de que algo possa ir mal ${ }^{(6,7,19)}$.

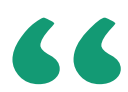

\section{Outro ponto a} ser discutido é a repulsa do público do sexo masculino em se exporem ao médico, justificando que mostrar parte do corpo ou ser tocado em um procedimento colocaria sua masculinidade em risco

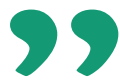

Dessa maneira, na tentativa de inserir melhor os homens no sistema de saúde e reduzir o número de morbimortalidade de pessoas do sexo masculino, foi for- malizada em agosto de 2009 a Política Nacional de Atenção Integral à Saúde do Homem (PNAISH), que enfrenta obstáculos, como o despreparo dos profissionais de saúde frente às peculiaridades desse grupo, evidenciando que a equipe de saúde também deve adaptar as estratégias de cuidado a realidade da masculinidade presente $^{(22,23)}$. É fundamental que se discuta sobre as masculinidades dentro dos serviços de saúde com os profissionais atuantes, bem como para a população, na tentativa de romper com o paradigma de invulnerabilidade dos homens ${ }^{(13-19,23)}$.

A próstata e o câncer: importância da prevenção

A próstata é um pequeno órgão pertencente ao grupo de glândulas sexuais acessórias do sistema reprodutor masculino e sua função consiste em secretar o líquido prostático, que além de compor $25 \%$ do sêmen, é formado por diversas enzimas proteolíticas como o PSA (muito utilizado na identificação do (AP), além de outras substâncias fundamentais para a produção de energia e sobrevivência dos espermatozóides ${ }^{(24)}$. Entretanto, doenças podem acometer tal órgão, como por exemplo, o CAP, que se dá por meio da multiplicação descontrolada de células do tecido que, posteriormente, originam metástases capazes de invadir órgãos vizinhos e se espalharem pelo corpo através dos sistemas linfático e sanguíneo ${ }^{(25)}$.

De acordo com o INCA, atualmente o CAP é considerado a segunda causa de óbito devido a neoplasias em homens, estando superado apenas pelo CPNM. Ainda segundo o INCA, espera-se que no período de 2020 a 2022 ocorram mais 65.840 casos novos de CAP $^{(26)}$. O CAP pode se desenvolver em qualquer indivíduo do sexo masculino, contudo, a existência de vários outros fatores de risco como a idade, o histórico familiar e hábitos de vida influenciam de forma direta no surgimento e evolução da patologia. O câncer pode assumir causas multifatoriais que contribuem para o seu desenvolvimento de modo a envolver fatores 
ambientais tais como o tabagismo, radiação ionizante, álcool e administração de hormônios; fatores endógenos como o envelhecimento, obesidade, alterações hormonais e a herança genética ${ }^{(20,22,23,27)}$.

Deste modo, o exame periódico com o urologista é de grande importância para a descoberta da doença em estágios iniciais e garantia de melhor eficácia do tratamento. Por isso, homens a partir dos 50 anos devem realizar anualmente, exames como a dosagem de PSA e o de toque retal. Porém, indivíduos com histórico familiar de CAP devem efetuar tais procedimentos a partir dos 40 anos, mesmo na ausência de sintomas ${ }^{(6-8,12)}$.

Principais métodos diagnósticos: PSA e exame de toque retal

O PSA é uma protease localizada no epitélio prostático e excretada no fluido seminal. Sua função principal é a liquefação do fluido seminal ${ }^{(28)}$. Até o início da puberdade, a próstata tem um crescimento lento e ao chegar nessa fase, a glândula aumenta o seu tamanho de maneira rápida se estabilizando logo em seguida até os 45 anos, quando tende a se expandir mais uma vez. Assim, a concentração de PSA, que só é produzido pelas células epiteliais da próstata, eleva-se com o aumento de volume da próstata e pode indicar infecção, hipertrofia benigna ou câncer $^{(4,5,24)}$.

Logo, a dosagem do PSA é um marcador tumoral ideal e por isso tem grande relevância, uma vez que sua produção é restrita à próstata. Todavia, se por um lado a análise laboratorial dessa glicoproteína tem grande importância para a garantia do diagnóstico do CAP, por outro, sua existência em altas quantidades também pode estar associada tanto à idade do paciente, como no caso da Hiperplasia Prostática Benigna (HPB) quanto a outros distúrbios na glândula como a prostatite e não somente ao câncer. Com exceção de valores extremos, o PSA não é suficientemente preciso para, de maneira isolada, estadiar o paciente ${ }^{(5,29)}$. Sendo assim, a dosagem do PSA deve ser solicitada pelo profissional médico como um exame complementar ao de toque retal e não como um método único para o diagnóstico.

Diante disso, nota-se que, embora o PSA tenha grande relevância para o diagnóstico, tratamento e monitoramento do CAP, sua utilização ainda gera controvérsias, já que em determinadas situações, sua presença no organismo em níveis maiores do que os esperados não determinam por si só a existência do CAP. Assim, evidencia-se a necessidade de mais estudos voltados à descoberta de outros marcadores tumorais específicos que sejam mais precisos e que auxiliem no diagnóstico do carcinoma prostático ${ }^{(4,5)}$.

O exame de toque retal é um método utilizado para avaliar estruturas localizadas internamente e próximas ao reto. Por meio dele, é possível efetuar o diagnóstico de diversas doenças na área, como o CAP. O procedimento é feito de forma rápida e indolor pelo profissional médico. Assim, o profissional introduz um dedo, utilizando luva e lubrificante no ânus do paciente e, dessa forma, apalpa a glândula com a finalidade de analisar o tamanho do órgão, a consistência e presença por possíveis tumores. Ademais, o toque retal é um método muito vantajoso, já que através dele é possível avaliar a próstata de forma instantânea, tem baixo custo, é feito em poucos minutos, não é invasivo e por isso não causa riscos ou seqüelas aos pacientes ${ }^{(6,8)}$.

Apesar de ser um procedimento proveitoso, o toque retal possui limitações e por isso necessita ser acompanhado de outros exames para obter o diagnóstico final, já que o tamanho da próstata pode estar associado a outros distúrbios na glândula como a Prostatite e a HPB. Também associada à técnica, existe o fato de que através dela, o profissional não consegue avaliar órgão como um todo. Sendo assim, o diagnóstico feito unicamente pelo toque retal não é garantia da presença de tumores malignos. Portanto, é essencial que seja associado à dosagem de PSA e, até mesmo à biópsia do tecido pertencente ao órgão. Este profissional se limita somente as partes posterior e lateral à palpação, ficando alguns tumores fora do alcance do examinador $r^{(4,5,7,8,10)}$.

Igualmente, o método também é vítima de muito preconceito pelos homens que associam o procedimento a uma violação de sua masculinidade. Por isso, é necessário o desenvolvimento de ações que objetivem conscientizar o público masculino acerca das consequências de tal pensamento ultrapassado para a saúde e do quanto é importante a descoberta da neoplasia prostática em estágios iniciais ${ }^{(6,7)}$.

\section{CAP: conduta médica e tratamento}

Após o diagnóstico de CAP, medidas são necessárias para evitar sua evolução. Segundo o Instituto Oncoguia, diversos tratamentos podem ser realizados, sendo que cada caso possui suas medidas terapêuticas necessárias, se levado em consideração o estágio em que a neoplasia se encontra. Resumidamente, podem ser realizadas cirurgias para a retirada dos tumores e a glândula, há também a possibilidade de efetuarem a quimioterapia e a radioterapia que, basicamente, objetivam inibir o crescimento das células cancerígenas destruindo-as. O transplante de medula óssea (TMO) também pode ser realizado quando associado aos outros métodos citados anteriormente(30).

Repercussões do CAP na masculinidade

Segundo o Ministério da Saúde (MS), em 2016, um estudo com mais de seis mil adultos do sexo masculino apontou que $1 / 3(31 \%)$ desse público ainda não têm o hábito de ir ao médico ${ }^{(31,32)}$. Uns dos principais motivos para essa ausência nos sistemas de saúde estão relacionados com a tentativa dos homens estarem sempre inseridos nos padrões de masculinidade hegemônica vigente ${ }^{(2,6,14)}$. Essa ausência no sistema de saúde contribui para menores ações de prevenção e para agravamentos, pois impede que os exames para diagnóstico do CAP aconteçam precocemente $^{(2,3,6,14,19,20,31)}$.

No que tange o CAP, no Brasil, a campanha chamando atenção para a 
neoplasia surgiu em 2008, promovida pelo Instituto Lado a Lado Pela Vida, uma Organização Não Governamental. Inicialmente chamada de "Um Toque, Um Drible", ela ganhou o nome "Novembro Azul" em 2012. Entretanto, mesmo com a campanha, concepções sociais impedem a procura da prevenção ou do diagnóstico precoce. Um dos motivos é o desconhecimento das formas de prevenção primária, secundária e de como é realizado tais procedimentos, o que gera receio e repulsa dos homens para realização dos exames $^{(33)}$. Além disso, outro motivo é o medo do diagnóstico porque, para muitos, saber que está possuindo o câncer é uma sentença de morte ${ }^{(6,8,13,14,16,19)}$.

Ademais, submeter ao exame de toque retal significa, para muitos homens, tocar na masculinidade, entre as justificativas do público do sexo masculino está a de que o exame toca em um espaço interdito do corpo. Igualmente, o toque retal pode simbolicamente ser visto como uma violação da masculinidade, uma vez que se associa à penetração sexual, passando a imagem do homem penetrador (ativo) para concepção de homem passivo no exame, assim ser passivo no momento do exame poderia contrariar o ideal de masculinidade. Outro fator é o temor de ter uma ereção durante o procedimento, pois a excitação poderia significar para o médico que o paciente está gostando, o que pode colocar em risco a sua masculinidade ${ }^{(6,10,13-20)}$.

Dessa forma, torna-se evidente como as concepções socioculturais acerca da masculinidade obstaculizam a realização do diagnóstico precoce do CAP e, conseqüentemente, contribuem para agravamentos e óbitos de pessoas do sexo masculino $^{(1-8,9,10,13-19)}$.
“

Dessa forma, tornase evidente como as concepções socioculturais acerca da masculinidade obstaculizam a realização do diagnóstico precoce do CAP e, conseqüentemente, contribuem para agravamentos e óbitos de pessoas do sexo masculino

\section{CONCLUSÃO}

Observa-se uma escassa procura do público masculino aos serviços de saúde. Tal ocorrência se deve principalmente às concepções socioculturais ultrapassadas que igualam o fato de estar doente à fragilidade, ideia que vai contra o conceito estabelecido do que é ser másculo na sociedade. Assim, tal pensamento retrógrado, implica em diversas consequências para a saúde masculina, visto que esse distanciamento dificulta a garantia de uma vida mais saudável.

Concomitantemente, no que se refere à busca pela prevenção do CAP, constata-se uma procura ainda menor pelos serviços de saúde, uma vez que, o medo de ser diagnosticado como doente aliado ao preconceito em relação ao exame de toque retal persiste de forma exacerbada. O problema é justificado, maiormente devido à forma de como o procedimento é realizado e como isso causa constrangimento, pois são recorrentes situações em que o exame de toque retal é visto como motivo de brincadeiras ofensivas em que, simbolicamente, o método é remetido a atos sexuais em que o homem é exposto à condição de passividade que vai contra a sua representação do que é ser viril e másculo na sociedade atual.

Assim, os casos de CAP possuem hoje números alarmantes e por isso é considerado um problema de saúde pública. Consequentemente, é notório que os maiores obstáculos dos serviços de saúde no que tange a saúde do homem e à prevenção do CAP, é o preconceito em relação ao exame de toque retal aliado a falta de preocupação desse público em cuidar da própria saúde além de, em certos casos, não aceitar o fato de que a possibilidade de estar doente possa de algum modo, fazê-lo se tornar mais frágil e vulnerável, já que tais atribuições são geralmente remetidas ao grupo feminino.

Portanto, torna-se imprescindível o desenvolvimento de ações em saúde que objetive conscientizar o público masculino acerca das consequências de tal pensamento ultrapassado para a garantia de uma vida saudável e do quanto é importante à realização de exames periódicos como o toque retal e a dosagem do PSA para a descoberta precoce do CAP. 1

\section{Referências}

1. Almeida MV. Gênero, masculinidade e poder: revendo um caso do sul de Portugal. In: Anuário Antropológico 95. Rio de Janeiro: Tempo Brasileiro; 1996.
2. Gomes R, Nascimento EF, Araújo FC. Por que os homens buscam menos os serviços de saúde do que as mulheres? As explicações de homens com baixa escolaridade e homens com ensino superior. Cad. Saúde Pú- 


\section{Referências}

blica [Internet]. 2007 [cited 2020 May 1];23(3):565-74. doi: https://doi. org/10.1590/s0102-311X2007000300015

3. Separavich MA, Canesqui AM. Saúde do homem e masculinidades na Política Nacional de Atenção Integral à Saúde do Homem: uma revisão bibliográfica. Saúde Soc. [Internet]. 2013 [cited 2020 May 1];22(2):415-28. doi: http://dx.doi.org/10.1590/S0104-12902013000200013

4. Amorim VMSL, Barros MBA, César CLG, Goldbaum M, Carandina L, Alves MCGP. Fatores associados à realização dos exames de rastreamento para o câncer de próstata: um estudo de base populacional. Cad. Saúde Pública [Internet]. 2011 [cited 2020 May 23];27(2):347-56. doi: https://doi. org/10.1590/S0102-311X2011000200016

5. Naoum S, Rocha MS. Marcadores tumorais utilizados no diagnóstico do câncer de próstata: antígeno prostático específico (PSA) e novas perspectivas. Rev. Acad. Oswaldo Cruz. 2016 [cited 2020 May 23];3(12):1-11. Available from: http://www.revista.oswaldocruz.br/Content/pdf/Edicao_12_ Naoum_Stephanie.pdf

6. Gomes R, Nascimento EF, Rebello LEFS, Araújo FC. As arranhaduras da masculinidade: uma discussão sobre o toque retal como medida de prevenção do câncer prostático. Ciênc. Saúde Colet. [Internet]. 2008 [cited 2021 Apr 11];13(6):1975-84. doi: https://doi.org/10.1590/S141381232008000600033

7. Gomes R, Rebello LEFS, Araújo $F C$, Nascimento EF. A prevenção do câncer de próstata: uma revisão da literatura. Ciênc. Saúde Colet. [Internet]. 2008 [cited 2021 Apr 11];13(1):235-46. doi: https://doi.org/10.1590/ S1413-81232008000100027

8. Souza LM, Silva MP, Pinheiro IS. Um toque na masculinidade: prevenção do câncer de próstata em gaúchos tradicionalistas. Rev. Gaúcha Enferm. [Internet]. 2011 [cited 2021 Apr 11];32(1):151-8. doi: http://doi. org/10.1590/s1983-14472011000100020

9. Pinto BK, Muniz RM, Schwartz E, Budó MLD, Lange RMHC. Identidade do homem resiliente no contexto do doente com câncer de próstata: uma perspectiva cultural. Rev. Bras. Enferm. [Internet]. 2014 [cited 2021 Apr 11];67(6):942-8. doi: http://doi.org/10.1590/0034-7167.2014670612

10. Araújo JS, Zago MMF. Masculinidades de sobreviventes de câncer de próstata: uma metassíntese qualitativa. Rev. Bras. Enferm. [Internet]. 2019 [cited 2021 Jan 2];72(1):231-40. doi: http://dx.doi.org/10.1590/00347167-2017-0730

11. Belinelo RGS, Almeida SM, Oliveira PP, Onofre PSC, Viegas SMF, Rodrigues $A B$. Exames de rastreamento para o câncer de próstata: vivência de homens. Esc. Anna Nery [Internet]. 2014 [cited 2021 Apr 11];18(4):697704. doi: https://doi.org/10.5935/1414-8145.20140099

12. Paiva EP, Motta MCS, Griep RH. Barreiras em relação aos exames de rastreamento do câncer de próstata. Rev. Latino-am. Enferm. [Internet]. 2011 [cited 2021 Jan 20];19(1):73-80. doi: http://dx.doi. org/10.1590/S0104-11692011000100011

13. Moraes MCL, Oliveira RC, Silva MJ. Uma questão masculina: conhecendo possíveis entraves para a realização dos exames de detecção do câncer de próstata. Rev. Med. Hered. [Internet]. 2017 [cited 2021 Feb 1];28(4):230-5. doi: http://doi.org/10.20453/rmh.v28i4.3222

14. Araújo JS, Nascimento LC, Zago MMF. Hegemonias corporificadas: dilemas morais no adoecimento pelo câncer de próstata. Rev. Esc. Enferm. USP [Internet]. 2019 [cited 2021 Abr 11];53:e03494. doi: http://dx.doi. org/10.1590/s1980-220x2018027403494

15. Araújo JS, Conceição VM, Zago MMF. Masculinidades transitórias no adoecimento pelo câncer de próstata. Rev. Latino-Am. Enferm. [Internet]. 2019 [cited 2021 Apr 11];27:e3224. doi: http://doi.org/10.1590/15188345.3248 .3224

16. Pinheiro TF, Couto MT, Silva GSN. Questões de sexualidade masculina na atenção primária à saúde: gênero e medicalização. Interface (Botucatu) [Internet]. 2011 [cited 2021 Jan 22];15(38):845-58. doi: http://doi. org/10.1590/S1414-32832011000300018

17. Gomes R. Sexualidade masculina e saúde do homem: proposta para uma discussão. Ciênc. Saúde Colet. [Internet]. 2003 [cited 2021 Feb
11];8(3):825-9. doi: http://dx.doi.org/10.1590/\$1413-81232003000300017 18. Astudillo MNM, Pinzón LAS, Ospina JJ, Grisales A, García JDR. Percepções sobre o câncer de próstata em povoação masculina maior de 45 anos. Santa Rosa de Cabral, 2010. Hacia Promoc. Salud [Internet]. 2011 [cited 2021 Jan 30];16(2):147-61. Available from: http://www.scielo.org.co/pdf/ hpsal/v16n2/v16n2a11.pdf

19. Silva PLN, Silva ELG, Santos VM, Galvão APFC, Oliveira VV, Alves CR. Motivação dos homens na busca por assistência prestada pelas estratégias de saúde da família. Nursing (São Paulo) [Internet]. 2021 [cited 2021 Apr 11];24(274):5377-88. doi: http://doi.org/10.36489/nursing. 2021v24i274p5377-5388

20. Silva SG. Masculinidade na história: a construção cultural da diferença entre os sexos. Psicol. Ciênc. Prof. [Internet]. 2000 [cited 2021 Jan 13];20(3):8-15. Available from: http://pepsic.bvsalud.org/pdf/pcp/v20n3/ v20n3a03.pdf

21. Gomes R. A construção social da masculinidade. Cad. Saúde Pública [Internet]. 2006 [cited 2020 Dez 2];22(5):1118. doi: http://dx.doi. org/10.1590/S0102-311X2006000500027

22. Coelho EBS, Schwarz E, Bolsoni CC, Conceição TB. Política Nacional de Atenção Integral à Saúde do Homem. Florianópolis: Universidade Federal de Santa Catarina, 2018 [cited 2021 Apr 12]. Available from: https://bvsms. saude.gov.br/bvs/publicacoes/politica_nacional_atencao_integral_saude homem.pdf

23. Cavalcanti JRD, Ferreira JA, Henriques AHB, Morais GSN, Trigueiro JVS, Torquato IMB. Assistência integral à saúde do homem: necessidades, obstáculos e estratégias de enfrentamento. Esc. Anna Nery. [Internet]. 2014 [cited 2020 Dez 15];18(4):628-34. doi: https://doi.org/10.5935/14148145.20140089

24. Tortora GJ, Nielsen MT. Princípios de anatomia humana. $12^{\mathrm{a}}$ ed. Rio de Janeiro: Guanabara Koogan, 2013.

25. Guyton AC, Hall JE. Tratado de fisiologia médica. $13^{\mathrm{a}}$ ed. Rio de Janeiro: Guanabara Koogan, 2017.

26. Brasil. Ministério da Saúde. Instituto Nacional do Câncer José Alencar Gomes da Silva. Estimativa 2020 - Incidência de câncer no Brasil. Rio de Janeiro: INCA, 2019 [cited 2020 Jun 26]. p. 33. Available from: http://inca. gov.br/sites/ufu.sti.inca.local/files/media/document/estimativa-2020-incidencia-de-cancer-no-brasil.pdf

27. Guerra MR, Gallo CVM, Mendonça GAS. Risco de câncer no Brasil: tendências e estudos epidemiológicos mais recentes. Rev. Bras. Cancerol. [Internet]. 2005 [cited 2020 Jun 26];51(3):227-34. Available from: https:// rbc.inca.gov.br/site/arquivos/n_51/v03/pdf/revisao1.pdf

28. Nardozza Júnior $A$, Zerati Filho $M$, Reis RB. Urologia Fundamental. $1^{a}$ ed. São Paulo: Planmark, 2010 [cited 2020 Jun 27]. Available from: http:// www.fcm.unicamp.br/fcm/sites/default/files/2016/page/urologia_fundamental.pdf

29. Almeida JRC, Pedrosa NL, Leite JB, Fleming TRP, Carvalho VH, Cardoso AAA. Marcadores tumorais: revisão de literatura. Rev. Bras. Cancerol. [Internet]. 2007 [cited 2020 Jun 27];53(3):305-16. Available from: http://rbc. inca.gov.br/site/arquivos/n_53/v03/pdf/revisao1.pdf

30. Instituto Oncoguia. Tratamentos do câncer. São Paulo: Instituto Oncoguia, 2013 [cited 2020 Jun 30]. Available from: http://www.oncoguia.org. $\mathrm{br} /$ conteudo/tratamentos/77/50/

31. Fundação Oswaldo Cruz. Instituto Gonçalo Moniz. Ministério da Saúde incentiva homens a cuidar da saúde. Salvador: Fiocruz Bahia, 2016 [cited 2020 Jun 28]. Available from: https://www.bahia.fiocruz.br/ministerio-da-saude-incentiva-homens-a-cuidar-da-saude/

32. Instituto Nacional de Câncer José Alencar Gomes da Silva. Câncer de próstata. Rio de Janeiro: INCA, 2021 [cited 2021 Mar 27]. Available from: https://www.inca.gov.br/tipos-de-cancer/cancer-de-prostata

33. Almeida ES, Souzas R, Dos-Santos EM. "Afectados por el tacto": sentidos atribuidos por hombres a las prácticas de prevención del cáncer de próstata. Salud Colectiva [Internet]. 2020 [cited 2021 Jan 19];16:e2176. doi: http://doi.org/10.18294/sc.2020.2176 